\title{
Front propagation in reaction-dispersal models with finite jump speed
}

\author{
Vicenç Méndez, ${ }^{1}$ Daniel Campos, ${ }^{2}$ and Sergei Fedotov ${ }^{3}$ \\ ${ }^{1}$ Departament de Medicina, Facultat de Ciències de la Salut, Universitat Internacional de Catalunya, c/ Josep Trueta s/n, E-08190 Sant \\ Cugat del Vallès (Barcelona) Spain \\ ${ }^{2}$ Grup de Física Estadística, Departament de Física, Universitat Autònoma de Barcelona, E-08193 Bellaterra (Barcelona) Spain \\ ${ }^{3}$ Department of Mathematics, University of Manchester Institute of Science and Technology (UMIST), Manchester M60 1DQ,
}

United Kingdom

(Received 23 March 2004; revised manuscript received 3 June 2004; published 29 September 2004)

\begin{abstract}
The continuous-time random walk and the Hamilton-Jacobi method are used to reach analytical expressions for the speed of traveling fronts in reaction-dispersal models. In this work the waiting time and jump length are assumed to be coupled random variables. The jump length for any jump is selected according to the waiting time at the end of the previous jump, and in consequence jumps of finite speed are performed. We study the effect of finite jump speed of the particles on the speed of the traveling fronts and find that in the parabolic and hyperbolic limits it can exceed the jump speed of the particles. We report analytical expressions for different probability distribution functions. Finally, we introduce the possibility that several particle speeds are allowed, so different dispersal mechanisms can be considered simultaneously.
\end{abstract}

DOI: 10.1103/PhysRevE.70.036121

PACS number(s): 02.50.Ey, 05.45.-a, 87.10.+e

\section{INTRODUCTION}

When both dispersal and generation (or annihilation) of particles determine the dynamics of a system, one has to deal with reaction-dispersal models. Specifically, one finds that when particles, initially standing in a finite domain, start spreading through a medium, wave-front solutions appear $[1,2]$, so looking for analytical expressions describing the front speed becomes then an essential objective.

One of the most common approaches to such dispersal models is the continuous-time random walk (CTRW) [3], which analyzes the evolution of a continuous probability $\rho(x, t)$ in space and time. However, most works based on this and others based directly on master equations usually consider that the times and distances covered by the particles are uncoupled; that is, particles, after a waiting time $\tau$, jump instantaneously from position $x$ to $x+\Delta x$, so they are assumed to have an infinite jump speed. In contrast with those works, in this paper we analyze the case of particles moving with a constant finite speed $v_{j}$, so time and distance jumps are coupled by means of the expression $\Delta x=v_{j} \tau$. This generalization has already been reported before by other authors [4] to study anomalous diffusion mechanisms. Here, we use the Hamilton-Jacobi method [5] to derive an expression for the wave-front speed appearing when $v_{j}$ is considered and we obtain that, for the parabolic and hyperbolic limits, the front speed may be higher than the jump speed of the particles. So the results obtained here will be of interest for real reaction-dispersal systems where the speed of the particles should be taken into account.

This paper is organized as follows. In Sec. I we present the general equations describing reaction-dispersal models in the CTRW framework and the Hamilton-Jacobi method leading to the front speed $v$. It is important to stress that the Hamilton-Jacobi method we will employ here holds only if the front evolves from an initial condition with compact support and if the growth function is concave. It is well known that for the case of parabolic reaction and diffusion, Aronson and Weinberger [6] showed that when the growth function is concave the front speed may be predicted by the linear marginally stability selection mechanism. Under this hypothesis, the Hamilton-Jacobi method will allow us to obtain the (linear) speed of the front. We study in Sec. II the case of jumps governed by the condition $\Delta x=v_{j} \tau$ and show that the restriction $v \leqslant v_{j}$ is satisfied for any complete probability distribution functions of waiting times and jump distances considered. In Sec. III, we generalize our analysis to the case when the particles are available to move with two (or more) jump speeds, every one with a certain probability. Finally, Sec. IV is devoted to final remarks and conclusions.

\section{REACTION-DISPERSAL MODELS}

We derive the evolution equation for the reactiondispersal process in one dimension according to the CTRW. The quantity that defines the motion is the probability distribution function (PDF) $\Psi(x, t)$ of a particle performing a jump of length $x$ after waiting a time $t$ at its starting point. If $P(x, t)$ is the probability density of arriving at point $x$ at time $t$ and $\rho(x, t)$ is the probability density of being at point $x$ at time $t$, we have

$$
\begin{aligned}
P(x, t)= & \int_{\mathbb{R}} d x^{\prime} \int_{0}^{t} d t^{\prime} \Psi\left(x-x^{\prime}, t-t^{\prime}\right) P\left(x^{\prime}, t^{\prime}\right) \\
& +P(x, t=0) \delta(t)+g(x, t), \\
& \rho(x, t)=\int_{0}^{t} d t^{\prime} \phi\left(t-t^{\prime}\right) P\left(x, t^{\prime}\right),
\end{aligned}
$$

where $\phi(t)$ is the probability of remaining at least a time $t$ on the point before proceeding with another jump. If $\varphi(t)$ $=\int d x \Psi(x, t)$ is defined as the waiting time PDF, by the definition of $\phi(t)$ one has 


$$
\phi(t)=\int_{t}^{\infty} d t^{\prime} \varphi\left(t^{\prime}\right)
$$

We have incorporated the initial condition $P(x, t=0)$ and $g(x, t)$ in a function accounting for the number density of new particles created at point $x$ at time $t$. The FourierLaplace transform of Eqs. (1) is

$$
\begin{gathered}
P(k, s)=\Psi(k, s) P(k, s)+P(k, 0)+g(k, s), \\
\rho(k, s)=P(k, s) \phi(s),
\end{gathered}
$$

so that

$$
\rho(k, s)=\frac{1-\varphi(s)}{s} \frac{f(k, s)}{1-\Psi(k, s)},
$$

where $f(k, s) \equiv P(k, 0)+g(k, s)$ and $s \phi(s)=1-\varphi(s)$ according to the Laplace transform of Eq. (2).

Now the jump length and waiting time are assumed to be coupled random variables where $\Phi(x)=\int_{0}^{\infty} d t \Psi(x, t)$ is the jump length PDF and $\varphi(t)=\int_{-\infty}^{\infty} d x \Psi(x, t)$, in contrast with the assumption used in most work, where the decoupled form $\Psi(x, t)=\Phi(x) \varphi(t)$ is introduced for simplicity. In a reaction-diffusion process the local growth function $f$ depends explicitly on $\rho$ as a nonlinear function and we will consider that $f$ is concave, that is, of the Fisher-KPP type [1,5]. Defining $f \equiv r \rho F(\rho)$ where $r$ is the constant growth rate, $F(\rho)$ must be such that $F(0)=1, F(\rho) \leqslant F(0)$, and $F(\rho)>0$ for $\rho \in(0,1)$.

To ensure an evolution with the minimal propagation speed we specify the initial condition with compact support

$$
\rho(x, 0)= \begin{cases}1, & x \leqslant 0 \\ 0, & x>0\end{cases}
$$

We assume that after a long enough time there exists a traveling wave solution to Eq. (1) under the above initial condition. Making use of the hyperbolic scaling $x \rightarrow x / \varepsilon$ and $t$ $\rightarrow t / \varepsilon$ we obtain a Hamilton-Jacobi equation which will be able to determine the position of the front and in consequence the speed of propagation. Under this scaling and the Fourier-Laplace antitransformed Eq. (3), the field $\rho^{\varepsilon}(x, t)$ $=\rho(x / \varepsilon, t / \varepsilon)$ obeys the equation

$$
\begin{aligned}
\rho^{\varepsilon}(x, t)= & \int_{0}^{t / \varepsilon} d t^{\prime} \int_{\mathbb{R}} d x^{\prime} \Psi\left(x^{\prime}, t^{\prime}\right) \rho^{\varepsilon}\left(x-\varepsilon x^{\prime}, t-\varepsilon t^{\prime}\right) \\
& +r \int_{0}^{t / \varepsilon} d t^{\prime} \phi\left(t^{\prime}\right) \rho^{\varepsilon}\left(x, t-\varepsilon t^{\prime}\right) F\left(\rho^{\varepsilon}\left(x, t-\varepsilon t^{\prime}\right)\right) .
\end{aligned}
$$

Inserting $\rho^{\varepsilon}(x, t)=\exp \left[-G^{\varepsilon}(x, t) / \varepsilon\right]$ into Eq. (4) one has

$$
\begin{aligned}
1= & \int_{0}^{t / \varepsilon} d t^{\prime} \int_{\mathrm{R}} d x^{\prime} \Psi\left(x^{\prime}, t^{\prime}\right) \\
& \times \exp \left[-\frac{G^{\varepsilon}\left(x-\varepsilon x^{\prime}, t-\varepsilon t^{\prime}\right)-G^{\varepsilon}(x, t)}{\varepsilon}\right] \\
& +r \int_{0}^{t / \varepsilon} d t^{\prime} \phi\left(t^{\prime}\right) \exp \left[-\frac{G^{\varepsilon}\left(x, t-\varepsilon t^{\prime}\right)-G^{\varepsilon}(x, t)}{\varepsilon}\right] \\
& \times F\left(-\frac{G^{\varepsilon}\left(x, t-\varepsilon t^{\prime}\right)-G^{\varepsilon}(x, t)}{\varepsilon}\right),
\end{aligned}
$$

and taking the limit $\varepsilon \rightarrow 0$ we have

$$
\begin{aligned}
1= & \int_{0}^{\infty} d t^{\prime} \int_{\mathbb{R}} d x^{\prime} \Psi\left(x^{\prime}, t^{\prime}\right) \exp \left(x^{\prime} \partial_{x} G+t^{\prime} \partial_{t} G\right) \\
& +r \int_{0}^{\infty} d t^{\prime} \phi\left(t^{\prime}\right) \exp \left(t^{\prime} \partial_{t} G\right),
\end{aligned}
$$

where $G(x, t)=\lim _{\varepsilon \rightarrow 0} G^{\varepsilon}(x, t)$. As long as the function $G(x, t)$ is positive, the rescaled field $\rho^{\varepsilon}(x, t) \rightarrow 0$ as $\varepsilon \rightarrow 0$. The boundary of the set where $G(x, t)>0$ can be regarded as a reaction-dispersal front. The Hamiltonian function and the conjugated momentum are defined by $H=-\partial_{t} G$ and $p=\partial_{x} G$, respectively; so Eq. (5) becomes the Hamilton-Jacobi equation

$$
1=\hat{\Psi}(p, H)+\frac{r}{H}[1-\hat{\varphi}(H)]
$$

where $\hat{\varphi}(H)=\int_{0}^{\infty} \varphi(t) e^{-H t} d t$ and

$$
\hat{\Psi}(p, H)=\int_{0}^{\infty} e^{-H t} d t \int_{\mathbb{R}} d x \Psi(x, t) e^{p x}
$$

is nothing but the bilateral-Laplace transform of $\Psi(x, t)$. The position of the front is determined by the equation $G(x, t)$ $=0$ so that $d G=\partial_{x} G d x+\partial_{t} G d t=p d x-H d t=0$ and the speed of the front will be $v=d x / d t=H / p$ [5,7]. On the other hand, $G(x, t)$ plays the role of the action functional and its solution is given in terms of the Lagrangian function $L=p d x / d s-H$ where $s$ is the temporal coordinate. The Hamilton equation $d x / d s=\partial H / \partial p$ allows us to compute $G(x, t)=\int_{0}^{t} L d s$ $=p(\partial H / \partial p) t-H t$ and from the condition $G(x, t)=0$ one finally has $\partial H / \partial p=H / p$. Therefore, the speed of the front is determined by the system of algebraic equations

$$
v=\frac{H}{p}, \frac{d H}{d p}=\frac{H}{p} .
$$

The latter is nothing but the existence condition for a minimum speed. So the Hamilton-Jacobi method predicts, if $f$ is concave and $\rho(x, 0)$ has compact support, that the speed selected by the front among all the mathematically possible values is that which reaches a minimum, in accordance with alternative approaches to the marginal stability [8].

\section{MODELS WITH A SINGLE JUMP SPEED}

The PDF $\Psi(x, t)$ may be written without loss of generality as $\Psi(x, t)=p(x \mid t) \varphi(t)$, where $p(x \mid t)$ is the conditional prob- 


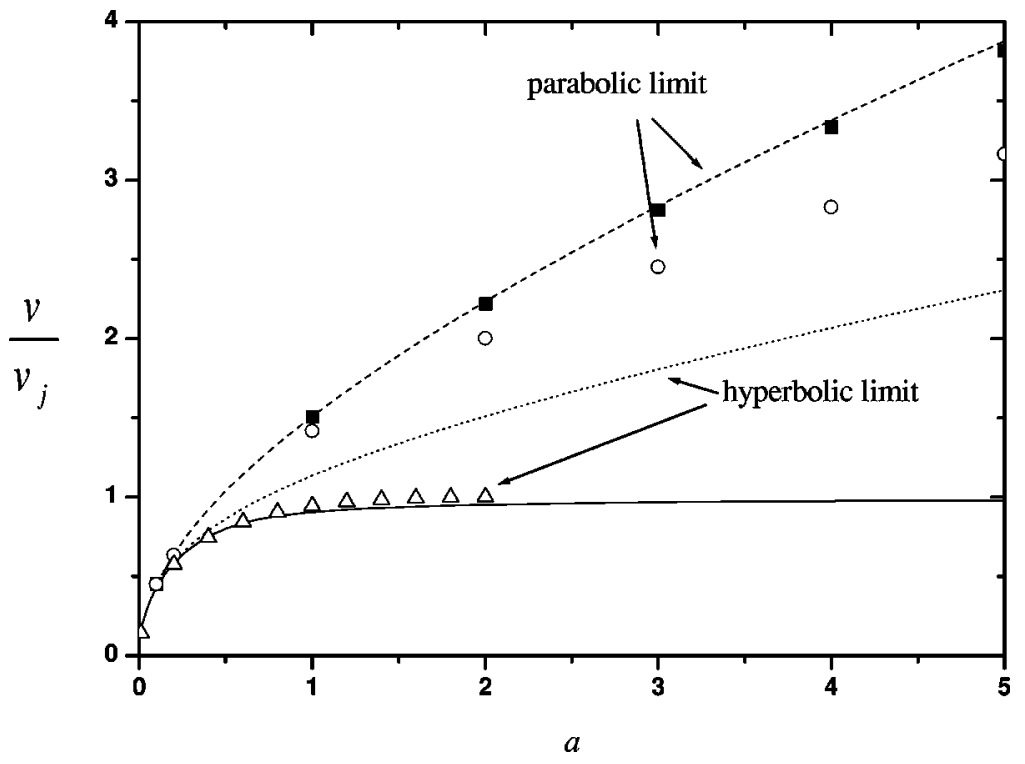

FIG. 1. Plot of the dimensionless front speed $v / v_{j}$ versus the dimensionless parameter $a$ given by Eq. (14) (solid line: This case corresponds to the complete waiting time PDF and complete jump length PDF); Eq. (16) (dashed line: This case corresponds to the first order waiting time PDF and complete jump length PDF); Eq. (17) (circles: This is the case of the Fisher speed, that is, first order in the waiting time PDF and second order in the jump length PDF); Eq. (19) (squares: This corresponds to first order in the waiting time PDF and fourth order in the jump length PDF); Eq. (20) (dots: This case corresponds to the second order waiting time PDF and complete jump length PDF); and Eq. (21) (triangles: This case corresponds to the second order in both the waiting time PDF and jump length PDF).

$$
\left(1-\frac{a}{H \tau}\right)[1-\hat{\varphi}(H)]>0,
$$

ability to move a distance $x$ in time $t$ [4]. We assume that particles perform isotropic jumps of finite velocity $\pm v_{j}$ [4]. When a particle waits a time $t$ in order to perform the next jump then it will have a length $x=v_{j} t$ and a duration $t$. So we take $p(x \mid t)=\frac{1}{2}\left[\delta\left(x-v_{j} t\right)+\delta\left(x+v_{j} t\right)\right]$ and

$$
\Psi(x, t)=\frac{1}{2}\left[\delta\left(x-v_{j} t\right)+\delta\left(x+v_{j} t\right)\right] \varphi(t) .
$$

Alternatively, one may write Eq. (9) as

$$
\Psi(x, t)=v_{j} \Phi(x)\left[\delta\left(x-v_{j} t\right)+\delta\left(x+v_{j} t\right)\right]
$$

if $\Phi(x)=\left(12 v_{j}\right) \varphi\left(|x| v_{j}\right)$, according to our arguments above. Performing the bilateral-Laplace transform of Eq. (9) one has

$$
\begin{aligned}
\hat{\Psi}(p, H) & =\int_{0}^{\infty} e^{-H t} \varphi(t) d t \int_{-\infty}^{\infty} d x p(x \mid t) e^{p x} \\
& =\left.\frac{1}{2} \varphi(s)\right|_{s=H-p v_{j}}+\left.\frac{1}{2} \varphi(s)\right|_{s=H+p v_{j}} .
\end{aligned}
$$

We show in turn that a strong condition which must be satisfied in the Hamilton-Jacobi equation is $H \tau>a$ where $a$ $=r \tau$ is a reaction-dispersal dimensionless number which is nothing but the quotient between two time scales: the characteristic jump time and the characteristic reaction time. To show this we start from the definition of $\hat{\Psi}(p, H)$ in Eq. (11):

$$
\begin{aligned}
\hat{\Psi}(p, H) & =\int_{0}^{\infty} e^{-H t} \varphi(t) d t \int_{-\infty}^{\infty} d x \frac{1}{2}\left[\delta\left(x-v_{j} t\right)+\delta\left(x+v_{j} t\right)\right] e^{p x} \\
& =\int_{0}^{\infty} e^{-H t} \varphi(t) \cos h\left(p v_{j} t\right) d t \\
& >\inf _{t \in[0, \infty)}\left[\cos h\left(p v_{j} t\right)\right] \hat{\varphi}(H) \\
& =\hat{\varphi}(H) .
\end{aligned}
$$

From Eqs. (6) and (12) one may write which is true if and only if $H \tau>a$. This condition, which has already been analyzed for the Hamilton-Jacobi method in [7], is noteworthy since it may restrict the range of values of $a$ where wave-front solutions can be found.

We perform now the study of the speed of fronts for two specific waiting time PDFs: the single waiting time PDF and the exponential PDF.

\section{A. Dirac $\delta$ waiting time PDF}

We assume $\varphi(t)=\delta(t-\tau)$; then, the particle dynamics is very simple: each particle waits a time $\tau$ before starting the next jump and this will be the time of jump duration. Thus, all jumps have finite velocity $v_{j}$ and length $\alpha=v_{j} \tau$. By the Laplace transform we have $\varphi(s)=e^{-s \tau}$ and from Eq. (11) one can obtain

$$
\hat{\Psi}(p, H)=e^{-H \tau} \cosh (\alpha p),
$$

which when introduced into the Hamilton-Jacobi equation (6) leads us to

$$
e^{H \tau}\left(1-\frac{a}{H \tau}\right)+\frac{a}{H \tau}=\cosh (\alpha p) .
$$

Likewise, from the first equation in (8)

$$
v=v_{j} \min _{z>a} \frac{z}{\cosh ^{-1}\left[e^{z}(1-a / z)+a / z\right]},
$$

where $z=H \tau$. It is interesting to note that Eq. (13) could also be obtained assuming decoupled jump lengths and waiting times and taking $\Phi(x)=\frac{1}{2}\left[\delta\left(x-v_{j} t\right)+\delta\left(x+v_{j} t\right)\right]$ and $\varphi(t)$ $=\delta(t-\tau)$, respectively. The minimum in Eq. (14) must be computed numerically for some specific values of $a$. When the characteristic jump time is large the particles jump long distances and the front travels faster. Moreover, if the characteristic reaction time is small the reaction process is fast 
and the front also propagates more rapidly. When both situations take place at the same time then $a \gg 1$ and one should expect a front traveling faster than for $a \ll 1$. In Fig. 1 we plot $v / v_{j}$ versus $a$ obtained from Eq. (14) and see that the speed of the front is a monotonically increasing function of $a$ but it is always lower than the jump velocity of the particles, as it should be from a physical point of view.

Dividing Eq. (3) by $\varphi(s)=e^{-s \tau}$ and taking the FourierLaplace transform of Eq. (9) with $\varphi(t)=\delta(t-\tau)$ one rewrites Eq. (3) as

$$
\left[e^{s \tau}-\cos \left(k v_{j} \tau\right)\right] \rho(k, s)=\frac{e^{s \tau}-1}{s} f(k, s) .
$$

In order to get an analytic result for the speed of the front $\hat{\varphi}(H)^{-1}$ is frequently approximated in a Taylor series for small waiting times [7,9]. For example, in the parabolic limit $\hat{\varphi}(H)^{-1}=e^{H \tau} \simeq 1+H \tau$ from Eq. (6) one has $H \tau=\cosh (\alpha p)$ $-1+a$ and from Eq. (8)

$$
v=v_{j} \min _{y>0} \frac{\cosh (y)-1+a}{y},
$$

where $y \equiv \alpha p$. Moreover, if one assumes short jump distances one may take the Taylor series of $\cosh (y)$ up to the second order, $\cosh (y) \simeq 1+y^{2} / 2$. This case corresponds to taking $e^{s \tau} \simeq 1+s \tau$ and $\cos \left(k v_{j} \tau\right) \simeq 1-\alpha^{2} k^{2} / 2$ in Eq. (15) which may be inverted by Fourier-Laplace transform to obtain the reaction-diffusion equation in the parabolic limit $\rho_{t}$ $=\left(\alpha^{2} / 2 \tau\right) \rho_{x x}+f(\rho)$ for which front solutions travel with the well known speed $v=v_{j} \sqrt{2 a}$. If one considers the above approximations $\hat{\varphi}(H)^{-1}=e^{H \tau} \simeq 1+H \tau$ and $\cosh (y) \simeq 1+y^{2} / 2$ in Eq. (14) one recovers the Fisher speed

$$
v=v_{j} \sqrt{2 a},
$$

and comparing with the Fisher speed $2 \sqrt{r D}$, the diffusion coefficient may be identified as

$$
D=\frac{v_{j}^{2} \tau}{2}
$$

If we take now $\cosh (y) \simeq 1+y^{2} / 2+y^{4} / 4$ ! in Eq. (16) then one gets

$$
v=v_{j} \frac{\sqrt{2}}{3} \frac{2 a-1+\sqrt{1+2 a}}{\sqrt{\sqrt{1+2 a}-1}} .
$$

Let us now show that the above behavior is corrected substantially when one adds the second order term in the expansion of $\hat{\varphi}(H)^{-1}$, that is, $\hat{\varphi}(H)^{-1}=e^{H \tau} \simeq 1+H \tau+(H \tau)^{2} / 2$, which corresponds to the hyperbolic limit [9]. From Eqs. (6) and (8) one obtains

$$
v=v_{j} \min _{y>0} \frac{\sqrt{(1+a / 2)^{2}+2 \cosh (y)-2}-1+a / 2}{y}
$$

which must also be computed numerically. If we take again $\cosh (y) \simeq 1+y^{2} / 2$ then the hyperbolic speed [9]

$$
v=v_{j} \frac{\sqrt{2 a}}{1+a / 2} \text { for } a<2
$$

is obtained. Certainly, taking $e^{s \tau} \simeq 1+s \tau+(s \tau)^{2} / 2$ and $\cos \left(k v_{j} \tau\right) \simeq 1-\alpha^{2} k^{2} / 2$ we may invert Eq. (15) by FourierLaplace to obtain $\tau \rho_{t t} / 2+\rho_{t}=\left(\alpha^{2} / 2 \tau\right) \rho_{x x}+f(\rho)+\tau f^{\prime}(\rho) \rho_{t}$ which has front solutions with a speed given by Eq. (21).

In Fig. 1 we plot $v / v_{j}$ for the following cases. The solid line is Eq. (14). This case corresponds to the complete waiting time PDF and complete jump length PDF. The dashed line shows Eq. (16). This case corresponds to the first order waiting time PDF and complete jump length PDF. The circles shows Eq. (17). This is the case of the Fisher speed, that is, first order in the waiting time PDF and second order in the jump length PDF. The squares are given by Eq. (19). This corresponds to first order in the waiting time PDF and fourth order in the jump length PDF. The dots represent Eq. (20). This case corresponds to the second order waiting time PDF and complete jump length PDF. Finally, the triangles shows Eq. (21). This case corresponds to the second order in both the waiting time PDF and jump length PDF. First of all it is interesting to note that in all the cases, the speed of the front is monotonically increasing with $a$, as one should expect. Second, some of the cases depicted are physically unrealistic. For example, all the parabolic limits may yield to fronts traveling with a speed higher than the particle jump speed. For the well known Fisher speed given by Eq. (17) only for $a<1 / 2$ has the result physical meaning. So, we and affirm that the parabolic limit has no physical meaning, unless $a$ is small enough. Regarding the hyperbolic limit only the second order approach in the jump length PDF yields a speed for fronts with physical meaning, but the analytic result given in Eq. (21) holds only for $a<2$. In conclusion, the hyperbolic limit, as well as the parabolic limit, has no physical meaning, unless $a$ is small enough. Finally, the complete waiting time and jump length PDFs yield a speed saturating to $v_{j}$ in the limit $a \rightarrow \infty$.

These results are of special interest since they make evident the importance of $v_{j}$ when one has to deal with real systems and a complete expression for the waiting time and jump length PDFs must be taken into account. It is also clear that $v_{j}$ should be known in order to achieve a realistic modelization.

\section{B. Exponential waiting time PDF}

We take now $\varphi(t)=\tau^{-1} e^{-t / \tau}$. From Eq. (11) one has

$$
\hat{\Psi}(p, H)=\frac{1+H \tau}{(1+H \tau)^{2}-\alpha^{2} p^{2}}
$$

and so, from Eq. (6),

$$
\frac{(1+H \tau)^{2}}{(1+H \tau)^{2}-\alpha^{2} p^{2}}=1+H \tau-a .
$$

If one retains the two lowest orders in a series expansion of the left hand side term in Eq. (22) for $H \tau \ll 1$ and $\alpha p \ll 1$ one has $H \tau=a+\alpha^{2} p^{2}$ which corresponds to the parabolic limit (Fisher's equation), and the speed of the front is $v=2 v_{j} \sqrt{a}$ which has physical meaning only for $a<1 / 4$. Comparing to 
the known Fisher speed $v=2 \sqrt{r D}$, one may define the diffusion coefficient

$$
D=v_{j}^{2} \tau,
$$

which is different from that in the case of a Dirac $\delta$ waiting time PDF [see Eq. (18)].

However, the speed of fronts for the complete waiting time and jump length PDFs may be obtained too. From Eqs. (8) and (22) it is computed as

$$
v=v_{j} \min _{y>a} \frac{y}{y+1} \sqrt{\frac{y+1-a}{y-a}},
$$

where $y=1+H \tau$. The minimum in Eq. (24) is a solution of $y^{2}-y(1+4 a)+2 a(1+a)=0$. The negative solution of this equation yields negative speeds and we retain only the positive one, which may be introduced in Eq. (24) to obtain

$$
v=v_{j} \frac{\left(4 a-1+\sqrt{1+8 a^{2}}\right)\left[\left(2 a-1+\sqrt{1+8 a^{2}}\right)\left(2 a+1+\sqrt{1+8 a^{2}}\right)\right]^{1 / 2}}{\left(2 a-1+\sqrt{1+8 a^{2}}\right)\left(4 a+1+\sqrt{1+8 a^{2}}\right)} .
$$

We observe in Eq. (25) a saturating behavior to $v=v_{j}$ when $a$ increases, which may be deduced by taking $a \rightarrow \infty$. Thus, the discussion made above about the saturation effect of introducing the jump velocity and the validity of Fisher and hyperbolic approximations still holds for the exponential waiting time PDF and it is expected to hold in general for any form of $\varphi(t)$.

\section{MODEL WITH TWO JUMP SPEEDS}

We study in this section the effect on the speed of the front if one considers that particles disperse according to two different jump velocities $v_{1}$ and $v_{2}$ with associated probabilities $\gamma_{1}$ and $\gamma_{2}$, respectively. This specific case is of great interest for systems involving two (or more) mechanisms of dispersal; some examples are the dispersion of seeds in ecology $[10,11]$ or the spread of epidemics $[1,12]$. Thus, we think that these fields can benefit from our work.

Analogously to Eq. (10), we have now

$$
\begin{aligned}
\Psi(x, t)= & \left(\frac{\gamma_{1}}{2}\left[\delta\left(x-v_{1} t\right)+\delta\left(x+v_{1} t\right)\right]\right. \\
& \left.+\frac{\gamma_{2}}{2}\left[\delta\left(x-v_{2} t\right)+\delta\left(x+v_{2} t\right)\right]\right) \varphi(t)
\end{aligned}
$$

with $\gamma_{1}+\gamma_{2}=1$. Taking the Laplace-bilateral transforms of Eq. (26) we reach

$$
\begin{aligned}
\Psi(p, H)= & \frac{\gamma_{1}}{2}\left(\left.\hat{\varphi}(s)\right|_{s=H-p v_{1}}+\left.\hat{\varphi}(s)\right|_{s=H+p v_{1}}\right)+\frac{\gamma_{2}}{2}\left(\left.\hat{\varphi}(s)\right|_{s=H-p v_{2}}\right. \\
& \left.+\left.\hat{\varphi}(s)\right|_{s=H+p v_{2}}\right] .
\end{aligned}
$$

By considering $\varphi(t)=\tau^{-1} e^{-t / \tau}$ in Eq. (27) and from Eq. (6) one has the following Hamilton-Jacobi equation:

$$
\frac{1+H \tau-a}{(1+H \tau)^{2}}=\frac{(1+H \tau)^{2}-\gamma_{1} v_{1}^{2} \tau^{2} p^{2}-\gamma_{2} v_{2}^{2} \tau^{2} p^{2}}{(1+H \tau)^{4}-\tau^{2}\left(v_{1}^{2}+v_{2}^{2}\right)(1+H \tau)^{2} p^{2}+v_{1}^{2} v_{2}^{2} \tau^{4} p^{4}} .
$$

Let us first consider some approximations to this model. If one expands the right-hand side of Eq. (28) up to second order in $\tau v_{i} p$ then the Hamilton-Jacobi equation (28) corresponds to the parabolic limit, it turns into $H \tau-a=\left(\gamma_{1} v_{1}^{2} \tau^{2}\right.$ $\left.+\gamma_{2} v_{2}^{2} \tau^{2}\right) p^{2}$, and the speed of the front is given by

$$
v=2 \sqrt{a\left(\gamma_{1} v_{1}^{2}+\gamma_{2} v_{2}^{2}\right)},
$$

where the diffusion coefficient may be defined by

$$
D=\left\langle v^{2}\right\rangle \tau=\left(\gamma_{1} v_{1}^{2}+\gamma_{2} v_{2}^{2}\right) \tau .
$$

We now work with the complete equation given in (28). From Eqs. (8) and (28) one has

$$
v=\sqrt{v_{1}^{2}+v_{2}^{2}} \min _{y>1+a} \frac{y-1}{\sqrt{z(y)}},
$$

where $y=1+H \tau$ and

$$
z(y)=y^{2} \frac{y-a-\beta-\sqrt{(y-a-\beta)^{2}-4 \eta(y-a)(y-a-1)}}{2 \eta(y-a)}
$$

with $\beta=\left(\gamma_{1} n^{2}+\gamma_{2}\right) /\left(n^{2}+1\right)$ and $\eta=n^{2} /\left(1+n^{2}\right)^{2}$ (where $n$ $\left.=v_{2} / v_{1}>1\right)$. In the limit $a \rightarrow \infty$, as $\beta<1$ for any value of $n$, then $y-a-\beta \simeq y-a$ and $y-a-1 \simeq y-a$. Therefore, one obtains $z(y) \simeq\left(n^{2}+1\right) y^{2} / n^{2}$ and Eq. (31) turns into

$$
v_{a \rightarrow \infty} \simeq \sqrt{v_{1}^{2}+v_{2}^{2}} \frac{n}{\sqrt{n^{2}+1}} \min _{y>1+a} \frac{y-1}{y}=v_{2} \frac{a}{a+1} \rightarrow v_{2} .
$$

So the speed of the front has an upper bound given by the highest jump speed of the particles, in accordance with the results and the physical restriction discussed in Sec. II for a single jump speed. In Fig. 2 we plot $v / v_{2}$ versus $a$ for different values of the probabilities $\gamma_{1}$ and $\gamma_{2}$. We observe also a saturating behavior (but in this case, toward the higher speed $v_{2}$ ) and see that the main effect of the probabilities $\gamma_{1}$ 


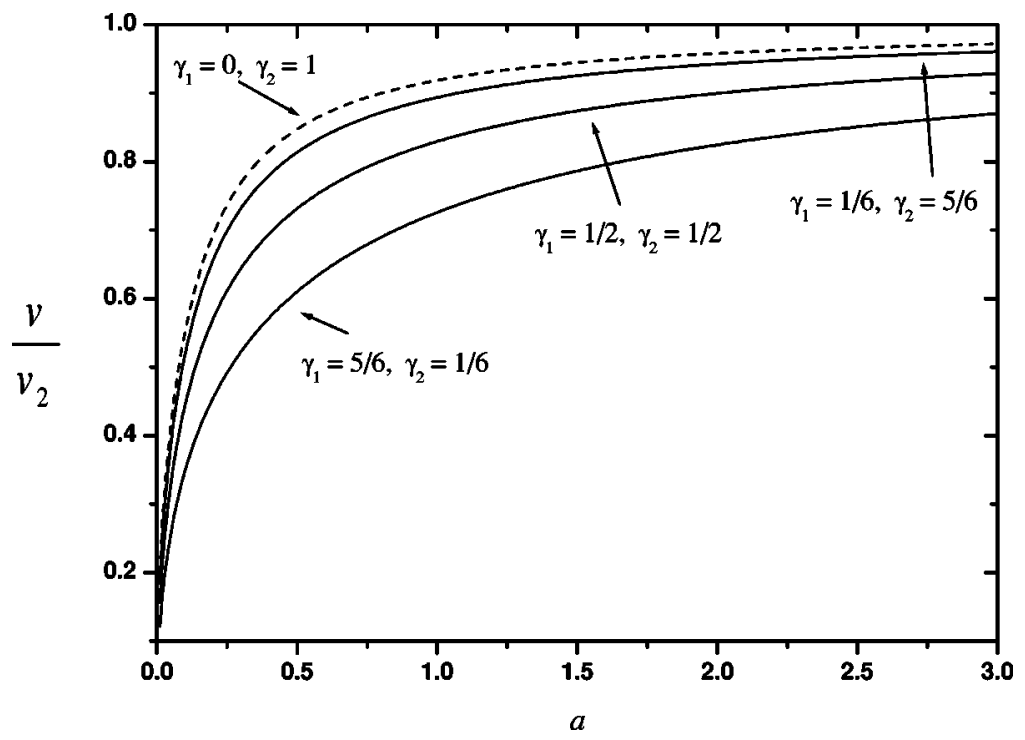

FIG. 2. Plot of the dimensionless front speed $v / v_{2}$ versus the dimensionless parameter $a$ given by Eq. (31) (solid lines) for different values of the probabilities $\gamma_{1}$ and $\gamma_{2}$. The dashed line corresponds to the case of a single jump speed given by Eq. (25) with $v_{j}=v_{2}$.

and $\gamma_{2}$ consists of controlling the saturation rate of $v$ as the parameter $a$ increases.

\section{CONCLUSIONS}

We have studied CTRWs modeling reaction-dispersal processes for the case of coupled waiting time and jump length random variables. Specifically, we have assumed that both are coupled in the form $\delta\left(x-v_{j} t\right)$ [see Eq. (9)], it is, by means of a single jump speed (Sec. II) or several jump speeds introducing the possibility of different mechanisms of dispersal (Sec. III). This coupling form has already been considered by some authors before [4] to study anomalous diffusion and, although more general expressions for $\Psi(x, t)$ could still be considered, we think that it is general enough to describe most real systems where dispersal processes are involved. Actually, the case of different jump speeds, every one occurring with a certain probability $\gamma_{i}$, could be generalized to the possibility of a normalized distribution of velocities $f(v)$. For that case, Eq. (9) would become

$$
\Psi(x, t)=\frac{1}{2}\left[\delta\left(x-v_{j} t\right)+\delta\left(x+v_{j} t\right)\right] \varphi(t) f\left(v_{j}\right),
$$

and the method leading to the expression of the front speed would be the same as shown in the previous sections. We have not used this generalized distribution here for the sake of clarity; we just mention that possibility to show that the assumption for $\delta\left(x-v_{j} t\right)$ can really describe a wide range of dispersal systems. Further generalizations could consider a dependence of jump velocities $v_{j}$ on $t$ or $x$; this possibility will be explored in future work.

As shown, the introduction of the jump speeds yields different qualitative behavior of the system which is studied here by means of the expression for the wave-front speed.
We have observed that parabolic and hyperbolic limits may yield nonphysical situations unless $a$ is small enough. Moreover, we observe that for the exact case (complete waiting time and jump length PDFs), $v$ saturates when the reaction parameter tends to infinity $(a \rightarrow \infty)$ toward the value of $v_{j}$, or toward the highest jump speed possible if several speeds are considered. In contrast with that behavior, the approximations reported here (parabolic and hyperbolic) taking small waiting times and distances of dispersal do not show this saturation (see Fig. 1). On seeing this, we conclude that classical reaction-diffusion equations $\left[\rho_{t}=\rho_{x x}+f(\rho)\right]$ and similar approaches which consider that walkers move instantaneously $\left(v_{j}=\infty\right)$ are in general not valid in the limit $a \rightarrow \infty$.

Finally, we stress that our work can be of interest for experimentalists working on reaction-dispersal systems, as the jump speed, the main parameter introduced here, can be frequently measured from experiments. For instance, we mention here the case of dispersal of seeds associated with the introduction of plants through new territories [10,11]. One of the main problems pointed out by the experts in this field is that dispersal kernels are difficult to measure and to deal with because many different vectors (wind, animals, gravity, etc.) take part in the dispersal process. The techniques presented here, where particles travel with one or more finite jump speeds, can offer an attractive framework for the study of such systems and many other behaving similarly.

\section{ACKNOWLEDGMENTS}

This work has been supported by the MCYT under Grants No. BFM 2003-06033 (V.M.) and No. REN-2003-00185 CLI (D.C.). 
[1] J. D. Murray, Mathematical Biology (Springer, Berlin, 1993).

[2] R. B. Banks, Growth and Diffusion Phenomena (Springer, Berlin, 1994).

[3] B. D. Hughes, Random Walks and Random Environments. Volume 1: Random Walks (Oxford University Press, New York, 1995).

[4] G. Zumofen and J. Klafter, Phys. Rev. E 47, 851 (1993)

[5] M. Freidlin, Functional Integration and Partial Differential Equations (Princeton University Press, Princeton, NJ, 1985).

[6] D. G. Aronson and H. F. Weinberger, Adv. Math. 30, 33
(1978)

[7] V. Méndez, D. Campos, and S. Fedotov (unpublished).

[8] W. van Saarlos, Phys. Rep. 386, 29 (2003).

[9] J. Fort and V. Méndez, Phys. Rev. Lett. 82, 867 (1999) Rep. Prog. Phys. 65, 895 (2002).

[10] R. Nathan et al., Nature (London) 418, 409 (2002).

[11] M. Williamson, Biological Invasions (Chapman and Hall, London, 1996).

[12] D. L. Smith et al., Proc. Natl. Acad. Sci. U.S.A. 99, 3668 (2002). 